\title{
Dual-comb imaging using soliton microcombs
}

\author{
Chengying Bao*, Myoung-Gyun Suh", and Kerry Vahala ${ }^{\dagger}$ \\ T. J. Watson Laboratory of Applied Physics, California Institute of Technology, Pasadena, CA 91125, USA \\ "These authors contributed equally \\ tvahala@caltech.edu
}

\begin{abstract}
We demonstrate rapid imaging using dual-microcomb interferometry. One of the soliton microcombs is spatially dispersed into two dimensions to record spatial information and the image is read out by multi-heterodyne with the second microcomb. @ 2019 The Author(s)
\end{abstract}

OCIS codes: (140.3945) Microcavities; (190.4390) Nonlinear optics, integrated optics.

The ability to record fast changing scenes is important to both fundamental studies and industrial production. Typical rapid imaging techniques rely upon table-top femtosecond lasers and CCD/CMOS detector arrays [1]. However, another more recent approach uses fast a single pixel photodetector combined with spatial multiplexing and can avoid certain challenges associated with array detectors [2]. In this approach, ultrashort optical pulses and their associated broadband spectra can be used for spatial multiplexing using spatial dispersers. For example, a grating and a virtual imaging phase array (VIPA) can act as a 2 dimensional (2D) disperser to disperse different optical frequencies into different spatial positions $[2,3]$. Thus, the object information can be encoded on the spectrum. Then, rapid imaging can be achieved by measuring the spectrum rapidly. For example, the encoded spectrum can be measured rapidly using the dispersive Fourier transform (DFT) technique. This method enables imaging with a $6 \mathrm{MHz}$ frame rate when using a mode-locked fiber laser as the optical pulse source [2]. In addition to dispersive Fourier transform, dual-comb interferometry can also be used for rapid spectral measurement [4]. Dual-comb interferometry based spectral retrieval has been used recently for spatial multiplexed imaging (termed dual-comb imaging in the following) by using the above mentioned 2D disperser [5]. In that demonstration, fiber laser based frequency combs were used. Due to the relatively low repetition rate of the fiber frequency combs $(100 \mathrm{MHz})$, the demonstrated frame rate was relatively low (12.34 Hz for 100 interferogram signals averaging). Here, we use $\mathrm{GHz}$ soliton microcombs generated from silica microresonators [6, 7] for rapid dual-comb imaging and a $200 \mathrm{kHz}$ frame rate is achieved. Although this frame rate is lower than the DFT based imaging retrieval method [2], it avoids the table-top fiber laser and the long dispersive fibers needed for DFT so that a miniature system on-a-chip is possible (see Fig. 1).

The experimental setup is illustrated in Fig. 1. Two silica wedge microresonators coupled via tapered fibers generate the microcombs used for the dual-comb imaging. Two types of microresonators with diameters of $7 \mathrm{~mm}$ and $36 \mathrm{~mm}$ are used, featuring repetition rates of $9.39 \mathrm{GHz}$ and $1.86 \mathrm{GHz}$, respectively [7]. One of the microcombs is dispersed into two dimensions by a VIPA and a grating. The VIPA disperses light vertically within its free-spectralrange (FSR, which is $60 \mathrm{GHz}$ in the experiments) which means that optical frequencies $v$ and $v+m f_{\mathrm{VIPA}}$ (where $m$ is an integer and $f_{\mathrm{VIPA}}$ is the VIPA FSR) will overlap in space. By adding a grating, frequencies $v$ and $v+m f_{\mathrm{VIPA}}$ can be horizontally dispersed to create a 2D spectral shower as illustrated in Fig. 1. The spectral shower is reflected by the object, coupled back into the fiber for return to a photodetector where it is heterodyned with the other microcomb with a slightly different repetition rate for image retrieval.

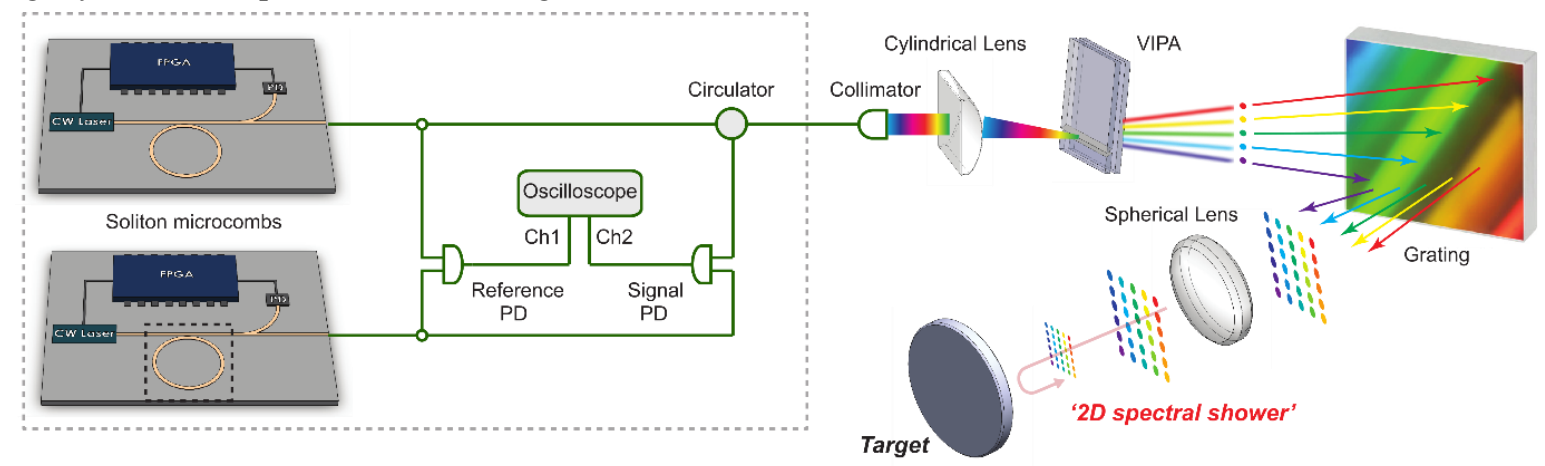

Fig. 1 Experimental setup for the dual-microcomb-imaging. Two on-chip silica microresonators are used to generate soliton microcombs for imaging. One of the microcombs is dispersed by a VIPA and a grating to create a 2D spectral shower. The spectral shower is focused on the target to encode object information on the spectrum. The encoded spectrum is then retrieved by heterodyning with the other comb having a slightly different repetition rate. 
To demonstrate the method, a USAF 1951 test target (negative) is imaged. In a first measurement, two silica microcombs with repetition rates close to $9.39 \mathrm{GHz}$ and a repetition rate difference of $1.5 \mathrm{MHz}$ are used. The power spectrum of one of the microcombs is shown in Fig. 2(a) and features a sech ${ }^{2}$-shaped spectral envelope.

(a)

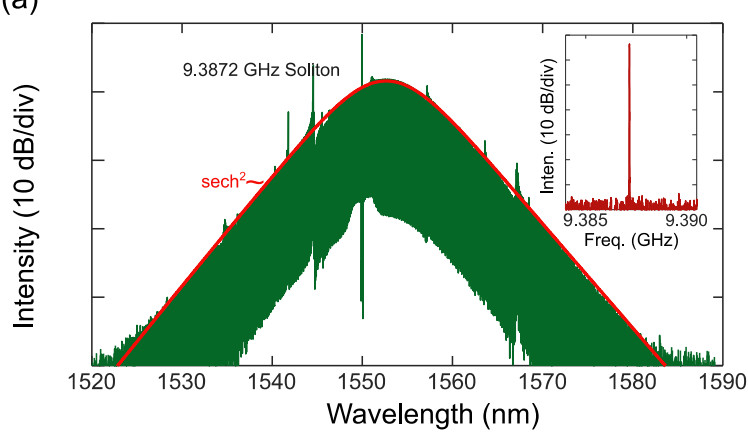

(b)
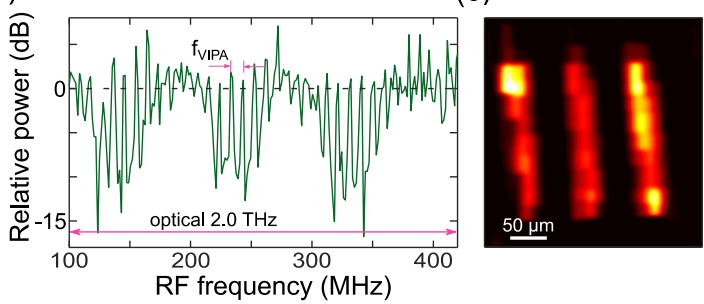

(d)

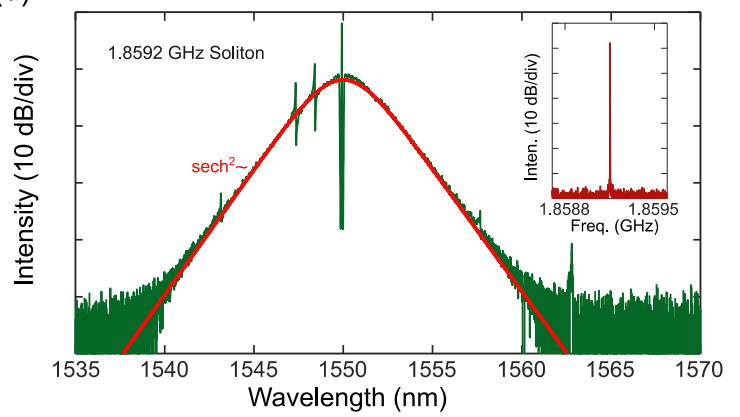

(e)

(f)

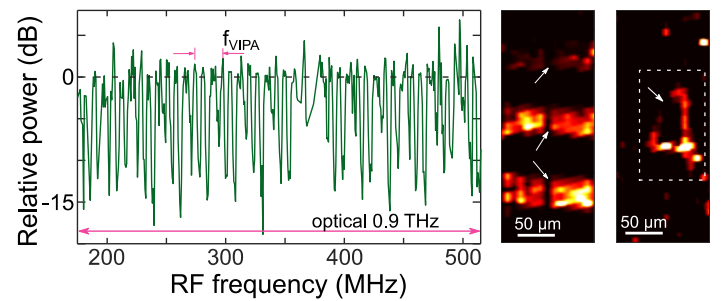

Fig. 2 (a) Optical spectrum of the $9.39 \mathrm{GHz}$ soliton microcombs showing a sech ${ }^{2}$ spectral envelope. The inset is the electrical spectrum of the photodetected soliton stream. (b) RF spectrum representing modulation from 3 vertical bars that are illuminated by the $9.39 \mathrm{GHz}$ microcomb. The integration time used to obtain the spectrum is $5 \mathrm{~s}$. (c) Image of three vertical bars reconstructed from the measured RF spectrum in (b). (d) Optical spectrum of the $1.86 \mathrm{GHz}$ soliton microcombs showing $\mathrm{sech}^{2} \mathrm{spectral}$ envelope. The inset shows the electrical spectrum of the photodetected soliton stream. (e) RF spectrum representing 3 horizontal bars illuminated by the $1.86 \mathrm{GHz}$ microcomb. (f) Reconstructed image from the spectrum in (e) showing 3 horizontal bars. (g) Image of number '4' on the USAF target produced using the $1.86 \mathrm{GHz}$ microcombs. The dark discontinuity shown by the arrows in ( $\mathrm{f}, \mathrm{g}$ ) results from the spectral notch produced by filtering the optical pump, see panel (d).

By recording the interferogram of the dual-comb and processing the signal in the frequency domain, we can retrieve the spectral modulation from the imaged target (Fig. 2(b)). This 1D spectrum is sorted into a 2D matrix with each column being one VIPA FSR to recover the image, as shown in Fig. 2(c). The integration time to obtain the spectrum in Fig. 2(b) is 5 s, i.e., a frame rate of $200 \mathrm{kHz}$ for imaging. In a second experiment, we use two $1.86 \mathrm{GHz}$ microcombs (see Fig. 2(d)) to increase the pixel number. The repetition rate difference between these two $1.86 \mathrm{GHz}$ microcombs is $0.7 \mathrm{MHz}$. A similar spectrum representing the target can be obtained in $10 \mathrm{~s}(100 \mathrm{kHz}$ frame rate) as shown in Fig. 2(e), and an image of 3 horizontal bars can be reconstructed (Fig. 2(f)). The $1.86 \mathrm{GHz}$ microcombs also enable resolution of more complex patterns, e.g., number '4' on the USAF1951 as shown in Fig. 2(g).

In conclusion, we have demonstrated rapid dual-comb imaging with a frame rate up to $200 \mathrm{kHz}$ using soliton microcombs. This fast imaging technique can find applications in life science. For example, it can be used in flow cytometry to monitor the flow of particles.

We acknowledge support from the Air Force Office of Scientific Research (FA9550-18-1-0353), the Kavli Nanoscience Institute and the Resnick Institute at Caltech.

\section{References}

1. J. Liang, and L. V. Wang. "Single-shot ultrafast optical imaging." Optica 5, 1113 (2018).

2. K. Goda, K. K. Tsia, and B. Jalali. "Serial time-encoded amplified imaging for real-time observation of fast dynamic phenomena." Nature 458, 1145 (2009).

3. V. Supradeepa, C. B. Huang, D. E. Leaird, and A. M. Weiner. "Femtosecond pulse shaping in two dimensions: Towards higher complexity optical waveforms". Opt. Express, 16, 11878 (2008).

4. I. Coddington, N. Newbury, and W. Swann. "Dual-comb spectroscopy." Optica 3, 414 (2016).

5. $\quad$ E. Hase, et al. T. Yasui, "Scan-less confocal phase imaging based on dual-comb microscopy." Optica 5, 634 (2018)

6. X. Yi, Q. F. Yang, K. Y. Yang, M. G. Suh, and K. Vahala, "Soliton frequency comb at microwave rates in a high-Q silica microresonator". Optica, 2, 1078 (2015).

7. M. G. Suh, and K. Vahala. "Gigahertz-repetition-rate soliton microcombs". Optica, 5, 65 (2018). 